\title{
Clinical and radiological features of cerebral amyloid angiopathy-related inflammation
}

\author{
Gary Álvarez Bravo ${ }^{1}$ [ $\cdot$ Laura Sánchez Cirera² . Lluís Ramió Torrentà ${ }^{2}$
}

Received: 15 March 2021 / Accepted: 15 July 2021 / Published online: 10 August 2021

(c) Fondazione Società Italiana di Neurologia 2021

\begin{abstract}
Objectives We want to report the clinical and radiological features of our cohort of patients diagnosed with cerebral amyloid angiopathy-related inflammation (CAA-RI) according to the Boston Criteria and additionally to disclose some atypical clinical characteristics observed in some of them to provide more knowledge about this novel entity.

Methods We describe 5 patients with probable CAA-RI according to a validation study of proposed criteria for the diagnosis of CAA-RI at University Hospital Josep Trueta of Girona. We consider some clinical characteristics which include the response to immunotherapy, CSF findings, and MRI features. The patient's neurologic outcomes were assessed using the modified Rankin Scale (mRS).

Results We collected 5 patients admitted for probable CAA-RI. Most were women and the median age was 72 years. The median $\mathrm{mRS}$ score at the onset of disease was 1 . Parietal lobes were most affected clinically as well as radiologically. Two patients had intracranial hemorrhage. Decreased levels of CSF amyloid beta 42 and 40 protein were observed. Corticosteroids were used in four patients and a remarkable improvement was observed in all of them.

Conclusions CAA-RI is a condition that predominantly affects parietal lobes according to our case series and this involvement seems to be directly related to a greater burden of microbleeds, cortical siderosis, WMH, and lobar hemorrhages on these lobes. Decreased levels of CSF amyloid beta protein plus increased total tau protein should be considered as part of the diagnostic criteria of CAA-RI. We recommend corticosteroids using, as they have been demonstrated to be very effective in managing CAA-RI.
\end{abstract}

Keyword VIAR-D-21-00,111

\section{Introduction}

Cerebral amyloid angiopathy-related inflammation (CAA-RI) is a recently recognized disease, which is characterized by perivascular inflammation due to an

Gary Álvarez Bravo

garinho29@icloud.com

Laura Sánchez Cirera

laura.13.sc@gmail.com

Lluís Ramió Torrentà

1lramio.girona.ics@gencat.cat

1 Unit of Neuroimmunology and Multiple Sclerosis of Girona, Department of Neurology, Hospital Universitari de Girona Doctor Josep Trueta, 10 Albereda Street, 17004 Girona, Spain

2 Department of Neurology, Hospital Universitari de Girona Doctor Josep Trueta, Girona, Spain autoantibody-mediated inflammatory response directed toward ${ }^{\circledR}$ amyloid.

Clinical manifestations include rapidly progressive dementia, epileptic seizures, and headaches. Neuroimaging is useful for a probable diagnosis, but neuropathology remains the gold standard in diagnosis. Treatment is based on immunosuppressive drugs.

\section{Methods}

\section{Data collection}

We describe 5 adult patients with probable CAA-RI that were diagnosed according to a validation study of proposed criteria for the diagnosis of CAA-related inflammation at University Hospital Josep Trueta (Girona, Spain) and the Unit of Neuroimmunology and Multiple Sclerosis of Girona, Catalonia-Spain. 


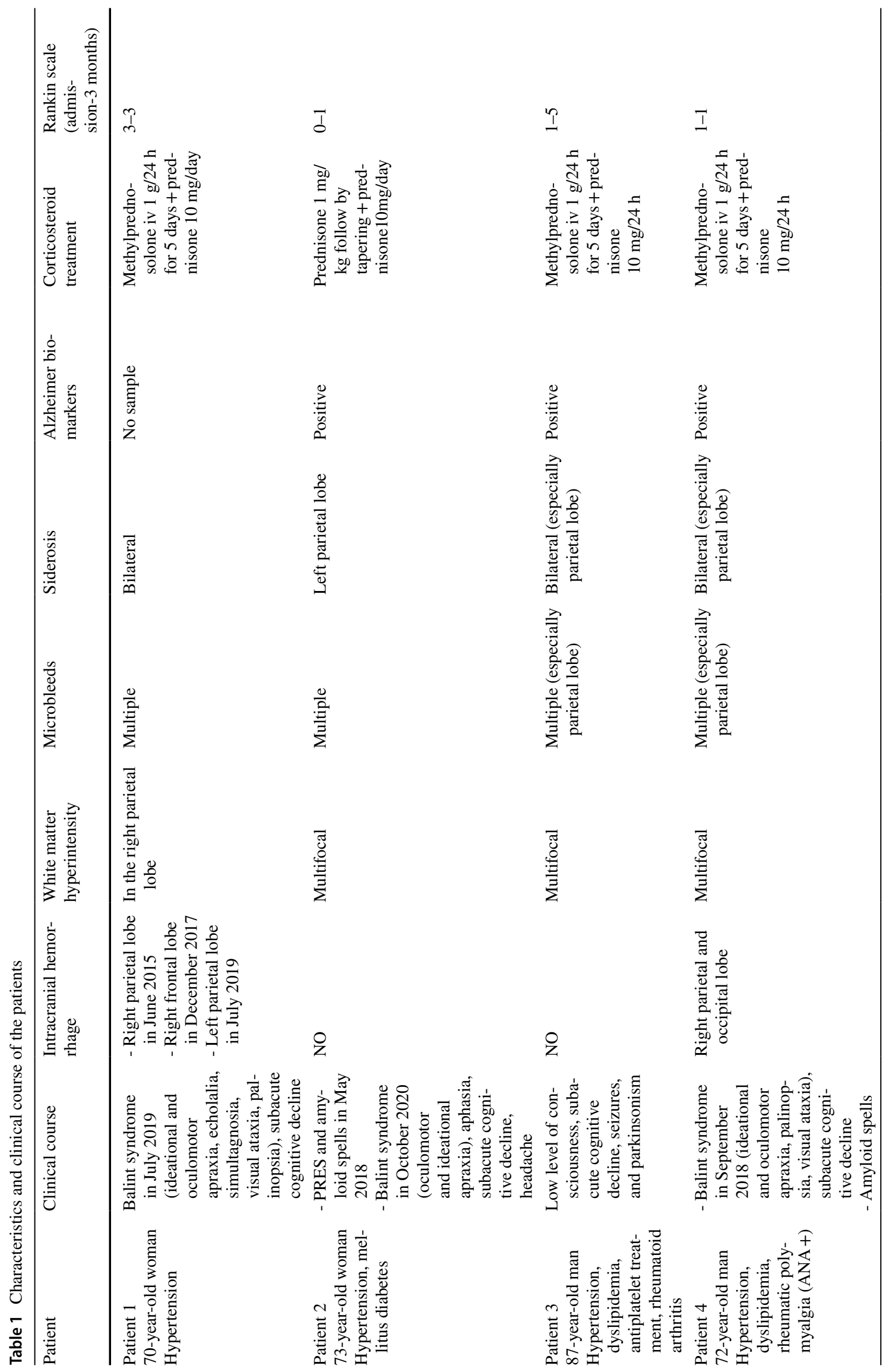




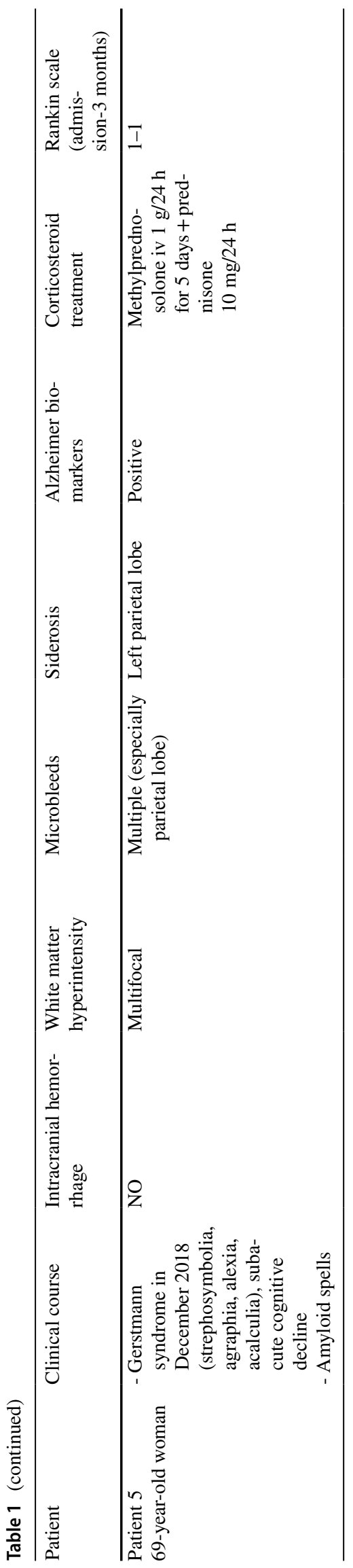

All the data presented here were collected during the course of clinical care. We focused on medical history, neurologic symptoms, characterization of symptoms by specific syndromes, MRI features, immunotherapy and its response, concomitant PRES, and neurodegeneration biomarkers on CSF. Patient neurologic outcomes were assessed using the modified Rankin Scale (mRS).

MRIs were performed and interpreted by the neuroradiologists.

\section{Standard protocol approvals, registrations, and patient consents}

We obtained written informed consent from all patients. The study was approved by the local institutional review boards of University Hospital Josep Trueta of Girona.

\section{Results}

The characteristics of the patients at baseline are summarized in Table 1. From August 2018 to December 2019, we evaluated 5 patients who were hospitalized due to neurological symptoms secondary to CAA-RI (Table 2). Three of them were women $(60 \%)$. The mean age was 74.2 (7.32) and the median mRS at the onset of disease: 1 [0-2]. Hypertension was a comorbidity more prevalent (80\%) and 3 patients $(60 \%)$ also had systemic pathology such as rheumatic polymyalgia.

Four patients (80\%) developed symptoms compatible with parietal lobe affection and three of them (75\%) had a Balint syndrome and one (25\%) had a Gerstmann syndrome. Four patients (80\%) had a subacute cognitive decline. Two patients had lobar hemorrhages (40\%), even patient 1 had a history of two intracranial hemorrhages prior to the last one in 2019.

Microbleeds were observed in all patients (100\%) and higher density was located in both parietal lobes, especially the left side.

Cortical siderosis was also observed in all patients (100\%) and was present in more than two lobes in three patients $(60 \%)$ while two patients $(40 \%)$ had it on the parietal lobes.

White matter hyperintensities (WMH) were observed in multiple locations in 4 patients $(80 \%)$ and one of them had it exclusively in the left parietal lobe exclusively.

One patient had meningeal enhancement (20\%) in the left parietal and temporal lobes. Three patients $(60 \%)$ had an early history of focal paroxysmal symptoms described as tingling or paresthesias. These symptoms were considered amyloid spells.

One patient who was hospitalized for seizures and acute cognitive impairment also had a 5-month history of parkinsonism without a recognized cause. 
Table 2 Absolute and relative frequencies of the main characteristics of the five patients

\begin{tabular}{|c|c|c|c|c|c|}
\hline & Absolute & Relative & & Absolute & Relative \\
\hline \multicolumn{6}{|l|}{ Gender } \\
\hline - Male & $2 / 5$ & $40 \%$ & Microbleeds & $5 / 5$ & $100 \%$ \\
\hline - Female & $3 / 5$ & $60 \%$ & & & \\
\hline \multicolumn{6}{|l|}{ Age } \\
\hline$->65$ & $5 / 5$ & $100 \%$ & PRES & $1 / 5$ & $20 \%$ \\
\hline$-\delta 65$ & $0 / 5$ & $0 \%$ & & & \\
\hline Hypertension & $4 / 5$ & $80 \%$ & Cortical siderosis & $5 / 5$ & $100 \%$ \\
\hline Dyslipidemia & $2 / 5$ & $40 \%$ & White matter hyperintensity & $5 / 5$ & $100 \%$ \\
\hline Mellitus diabetes & $1 / 5$ & $20 \%$ & Leptomeningeal enhancement & $1 / 5$ & $20 \%$ \\
\hline Anticoagulant treatment & $0 / 5$ & $0 \%$ & Amyloid spells & $3 / 5$ & $60 \%$ \\
\hline Systemic disease & $3 / 5$ & $60 \%$ & Alzheimer biomarkers & $4 / 4$ & $100 \%$ \\
\hline \multicolumn{6}{|l|}{ Clinical syndrome } \\
\hline - Parietal & $4 / 5$ & $80 \%$ & Methylprednisolone $1000 \mathrm{mg}$ & $4 / 5$ & $80 \%$ \\
\hline - Temporal & $1 / 5$ & $20 \%$ & & & \\
\hline Seizures & $1 / 5$ & $20 \%$ & Corticosteroids improvement & $4 / 4$ & $100 \%$ \\
\hline \multirow[t]{3}{*}{ Rapidly progressive dementia } & $2 / 5$ & $40 \%$ & Rankin pre-hospitalization & & \\
\hline & & & $-\delta 2$ & $4 / 5$ & $80 \%$ \\
\hline & & & $->2$ & $1 / 5$ & $20 \%$ \\
\hline \multirow[t]{3}{*}{ Intracranial hemorrhage } & $2 / 5$ & $40 \%$ & Ranking post-hospitalization & & \\
\hline & & & $-\delta 2$ & $3 / 5$ & $60 \%$ \\
\hline & & & $->2$ & $2 / 5$ & $40 \%$ \\
\hline
\end{tabular}

Neurodegeneration biomarkers on CSF were measured in 4 patients. All of them (100\%) had decreased levels of amyloid beta 42 and 40 protein and mildly increased total tau protein.

Methylprednisolone $1000 \mathrm{mg}$ was used in four patients $(80 \%)$ and one patient $(20 \%)$ received prednisone $1 \mathrm{mg} / \mathrm{kg}$, with a remarkable improvement in all of them. At 3 months, all patients were taking prednisone $10 \mathrm{mg} /$ daily after corticosteroid tapering. Their median of mRS was 1 [1-4].

\section{Discussion}

CAA is a vascular disease that can cause hemorrhagic, ischemic or leukoencephalopaty, and age-related cognitive decline. It is caused by a cerebrovascular deposition of amyloid- $\beta$. When there is a perivascular inflammatory reaction, CAA is known as CAA-RI [1].

Pantelakis raised the hypothesis that vascular amyloid may originate in the brain: (i) preferential involvement of small arterioles and capillaries of the leptomeninges and cerebral cortex, without necessarily involving adjacent parenchymal; (ii) topographical distribution favoring posterior lobar brain regions; (iii) lack of involvement of white matter small vessels; (iv) association with increased age and dementia; (v) lack of association with hypertension and arteriosclerosis; and (vi) lack of any link with systemic amyloidosis [2, 3]. We observed that posterior areas were also most involved but unlike Pantelakis report, parietal lobes were most affected in our case series, clinically as well as on MRIs. It has been shown that amyloid deposition is preferably located on vessels of posterior regions; based on this fact, in our case series, we noted the greatest burden of microbleeds, lobar hemorrhages, cortical siderosis, and involvement of the white matter was located in posterior regions. At the same time, this mechanism could be the reason why patient 2 developed PRES during the clinical course, as CAA has been recognized as the cause of this [4].

Typical clinical presentation is characterized by acute or subacute cognitive decline, seizures, headache, and focal neurological signs. Lobar hemorrhages (LH) are seen less commonly. Although a definitive diagnosis requires a brain biopsy, this procedure is done in few cases. There are criteria based on clinical and radiological features for the diagnosis of probable or possible CAA-RI [1]. None of our patients required a brain biopsy and all of them met the criteria for probable CAA-RI.

Despite the posterior lobes' involvement is well-recognized in CAA-RI, Balint and Gerstmann syndromes have not been described to be secondary to CAA-RI; thus, this case series is first on disclosing these entities as part of the clinical spectrum of CAA-RI. Patient 3 had seizures at the moment of diagnosis and had developed parkinsonism 5 months before his admission to the hospital, which improved during hospitalization after receiving treatment with steroids. In the third stage of CAA, amyloid deposition 
Fig. 1 MRI finding. Legend: A FLAIR sequence of MRI corresponding to a right parietal intracranial hemorrhage. B and $\mathbf{C}$ Presence of multilobar white matter hyperintensities in FLAIR sequences of MRI. D Left parietal cortical siderosis and right parietal intracranial hemorrhage in BOLD sequence of MRI. B Presence of multiple diffuse microbleeds and cortical siderosis in BOLD sequence of MRI. E and F Multiples microbleeds and cortical siderosis in BOLD sequence of MRI. $\mathbf{G}$ and $\mathbf{H}$ Left parietal meningeal enhancement in FLAIR sequence of MRI. I Multiple microbleeds in basal ganglia in BOLD sequence of MRI
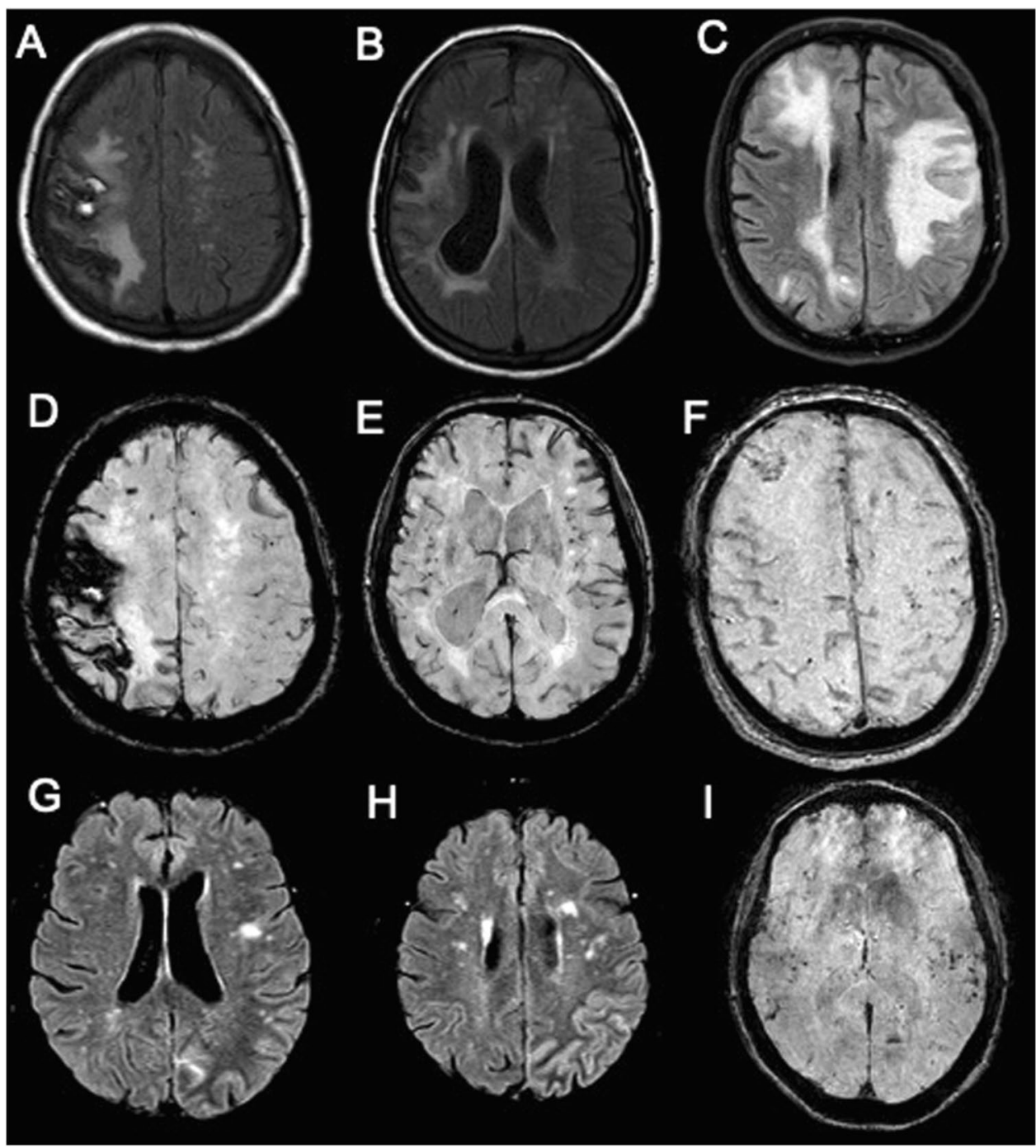

H
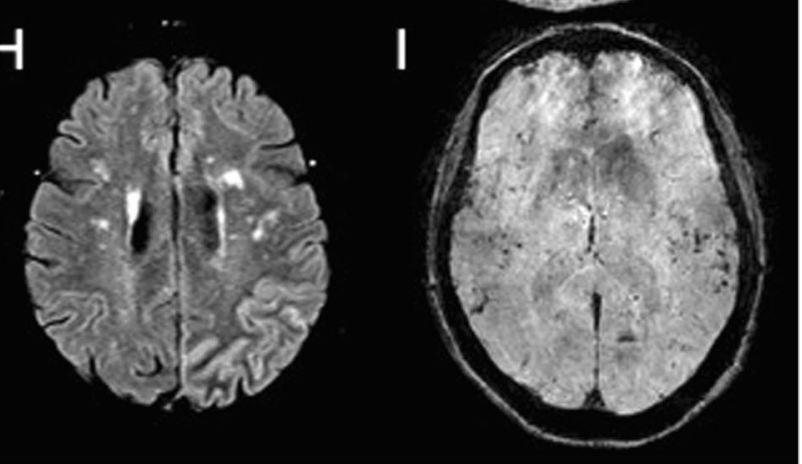

is observed in the basal ganglia, thalamus, and in the lower brainstem [5]. Due to this fact, we speculate that parkinsonism can be a late presentation of CAA-RI, and for this reason, its prevalence is underestimated. Recently, parkinsonism steroid-responsiveness secondary to CAA has been published [5].

During the work-up approach, there were two facts that caught our attention: patient 2 was positive in IgM for cytomegalovirus and patient 3 had an abrupt exacerbation of parkinsonism before dying due to COVID infection. We hypothesize that virus infections might trigger an inflammatory cascade in patients with CAA. Indeed, currently, there are ongoing studies to demonstrate a link between exacerbation of autoimmune disorders and concomitant infection by SARS-CoV-2 [6].

Cortical siderosis, microbleeds, $\mathrm{LH}$, and WMH are MRI findings needed to diagnose CAA-RI [7, 8]. All of them were observed in our patients (Fig. 1).
Parietal lobes were most affected by these alterations. Meningeal enhancement is another radiologic finding and was observed in one of our patients.

CAA remains clinically distinct from Alzheimer's disease (AD) but has the potential to link cerebrovascular and neurodegenerative pathways in the aging brain [9]. We could notice this link, because biomarkers on CSF used to support the clinical diagnosis of AD [7] showed a very similar profile in all our studied patients. Indeed, we suggest that decreased levels of CSF amyloid beta 42 and 40 protein plus increased total tau protein be included within diagnostic criteria of CAA-RI in a proper scenario [10]. Anti-amyloid beta antibodies in the CSF were not performed in our patients since they are not available in the current clinical practice.

Available evidence suggests that up to $70 \%$ of patients affected by CAA-RI may be responsive to immunosuppressive therapy, especially to glucocorticoids, but a consensus has not been reached about treatment during followup. Other immunosuppressive treatments that have been 
associated with a favorable response include cyclophosphamide, methotrexate, and mycophenolate mofetil [11]. Of 5 patients, 4 received $1000 \mathrm{mg}$ of methylprednisolone per day for 5 days followed by an oral steroid taper and all of them had a sustained clinical improvement measured by mRS at the moment of discharge from the hospital and 3 months later. Patient 2 did not receive treatment with megadoses of methylprednisolone but she was taking $1 \mathrm{mg} /$ $\mathrm{kg}$ of prednisone followed by tapering. Currently, all patients are taking $10 \mathrm{mg}$ of prednisone to prevent new intracranial hemorrhages.

\section{Declarations}

Ethical approval This study was approved by the Ethical Committee of The Hospital Universitari Dr. Josep Trueta of Girona, Spain.

Conflict of interest The authors declare no competing interests.

Informed consent None.

\section{References}

1. Auriel E, Charidimou A, Gurol ME et al (2016) Validation of clinicoradiological criteria for the diagnosis of cerebral amyloid angiopathy-related inflammation. JAMA Neurol 73:197

2. Pantelakis S (1954) A particular type of senile angiopathy of the central nervous system: congophilic angiopathy, topography ad frequency. Monatsschr Psychitr Neurol 128:219-256
3. Charidimou A, Bloulouis G, Gurol ME et al (2017) Emerging concepts in sporadic cerebral amyloid angiopathy. Brain 140:1829-1850

4. Lilleker J, Vassallo J, Punter M (2013) Reversible Leukoencephalopaty as a presentation of cerebral amyloid angiopathy. J Neurol Neurosurgery Psychiatry 84:e2

5. Yamashita Y, Hatano T, Ogawa T, Daida K, Motoi Y, Hattori N (2020) Steroid-responsive parkinsonism caused by cerebral amylod angiopathy-related inflammation. Mov Disord Clin Pract 7(3):329-331

6. Liu Y, Sawalha AH, Lu Q (2021) COVID-19 and autoimmune diseases. Curr Opin Rheumatol 33(2):155-162. https://doi.org/ 10.1097/BOR.0000000000000776

7. Boyano I, Bravo N, Miranda J, Gil-Gregorio P, Olazaran J (2018) Microhemorragias cerebrales: epidemiologia e implicaciones clínicas. Neurologia 33:515-525

8. Pongpitakmetha T, Fotiadis P, Pasi M et al (2020) Cortical superficial siderosis progression in cerebral amyloid angiopathy. Neurology $94: 1-\mathrm{e} 13$

9. Cordonnier C, Leys D, Dumont F et al (2010) What are the causes of pre-existing dementia in patients in patients with intracerebral haemorrhages? Brain 133:3281-3289

10. Renard D, Castelnovo G, Wacogne A (2012) Interest of CSF biomarkers analysis in possible cerebral amyloid angiopathy cases defined by the modified Boston criteria. J Neurol 259(11):2429

11. Eng JA, Frosch MP, Choi K, Bebeck GW (2004) Clinical manifestations of cerebral amyloid angiopathy-related inflammation. Ann Neurol 55(2):250

Publisher's note Springer Nature remains neutral with regard to jurisdictional claims in published maps and institutional affiliations. 